\title{
Social annotation tools in higher education: a preliminary systematic review
}

\begin{abstract}
This review discusses the evidences in using SA tools in higher education settings. Using a detailed inclusion/exclusion procedure, 71 studies were included. A large number of studies were centred on system design issues and the evaluation of designed tools within education and computer technology classes, with blended learning modality among undergraduates. Findings suggested there was a gradual increase in the frequency of SA-based publications, with Science Direct, Taylor \& Francis, and IEEE as the three databases with the most SA publication experiences. Findings were mostly derived from quasi-experiments. Of the four major topics recognised, 'system design and implementation issues' was categorised as the first topic, followed by 'the effectiveness of SA tools on process-oriented measures', 'the effectiveness of SA tools on outcome-oriented measures', and 'the improvement of SA tools and learning design'. Process-oriented and outcome-oriented measures dominating the studies were quantity and quality of annotations and reading performance, respectively.
\end{abstract}

Keyword: Social annotation tools; Annotation-based systems; Higher education; Anchored online discussions; Blended learning; Systematic review 